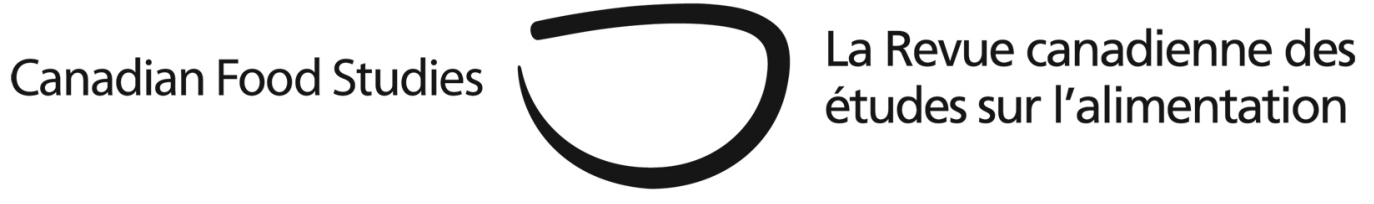

\title{
Editorial
}

\section{Inspiring and informing through food studies}

\author{
Ellen Desjardins
}

This issue offers a rich and diverse selection of material from Canadian authors and reviewers. It takes us across the country, highlighting particular communities where food-related transformation is happening, and it encourages us to think differently about technology and subjectivity. Notably, the methods used by several authors involve critically dissecting language - whether that be the discourse or terminology used by food systems change-makers or by people who are themselves affected by food system change.

Often, the ordinariness of familiar terms or concepts belies their complexity and hidden sides, necessitating closer scrutiny. "Big data" is one such phenomenon, upon which Bronson and Knezevic shine a critical spotlight. Showing how current data sources and data collection technologies differ from those of the past, the authors make the case that current big data are more than neutral numbers, but benefit productivist food regimes. They point to the need for research to document the consequences of big data to a broader group of food systems models. Another popular phenomenon is the "food charter": dozens of such manifestos have materialized across Canada in the past decade, signifying positive, united visions for the food systems of cities and regions. Or is that just one side of the coin? Spoel and Derkatch analyse the food charter as a "genre", examining their rhetoric and embedded ideologies. They suggest that charters perform not just by reflecting inherent values, but by aspiring to shape a food system in an uncontested way. The authors ask if charters may mask, rather than reveal, tensions and contradictions; with this in mind, they recommend that researchers evaluate the societal and policy fallout of charters, including both their intended actions as well as their exclusions and unintended effects.

MacLeod, in her Perspectives article, takes just such an approach, although without focusing on food charters. She brings us to Cape Breton Island, where she investigates changing "ecological” food practices among the islanders. Her information comes from discourse derived 
from ethnographic interviews, observations at events and markets, restaurant menus, and policy documents. What she finds there is not a clear, linear pathway of change, but divergent discourses in multiple arenas (personal, social, economic, dietary), revealing a tangled web of new power relations, identities, struggles and opportunities. With this in-depth work, MacLeod has provided baseline evidence upon which new projects and policies can be built. LavalléePicard offers us similar insights from her tale of two communities: Saint-Camille in Québec and Salt Spring Island in British Columbia. In what ways are these different populations moving towards food sovereignty? Answers are again sought in discourse, revealing the systemic challenges that people are struggling with, as well as the practices and circumstances that are nourishing positive change. Colatruglio and Slater bring more narrative into the picture, this time from young adults who have left home and are voicing the everyday experiences of preparing food for themselves. What emerges is a multi-faceted set of personal, environmental, and social factors that influence food literacy, or lack of it.

An unmistakable thread runs through these collective pieces that expose us to personcentred activities around food, eating, politics, and shifting food environments. Inevitably, it is messy and fascinating and dynamic. Here is where Szanto is waving madly at us from the sidelines, exhorting us to go a step further and acknowledge something that he argues is here already: the validity - indeed the honesty - to express subjectivity when we research and write about food. To ensure a normative yin/yang-like balance within food studies, rather than sticking with dispassionate objectivity, we support, in this journal, the inclusion of first-person narrative, reflexivity, and emotional dimensions in all types of submissions. Is there sufficient evidence for a personal turn in food-related literature, as Szanto suggests there might be? Is it hot in here? I feel we can make it so.

Finally, a word about event and book reviews. Tudge describes the BPLTC 111: Food Control artistic exhibition in Montréal so vividly-including images - that we can picture ourselves there, experiencing the avant-garde, bio-political representations that make three food system trends (fermentation, industrial mono-culture agriculture, and seed banking) come alive in novel ways. Additionally, the five book reviews, shepherded by associate editor Phil Mount, span a wide range of topics, including local food supply chains in Nebraska, market transformation strategies for sustainability, cheese-making and its politics, critiques of fat activism, and a deconstruction of gluten-bashing and other fads. All worth reading!

Regarding our editorial team, we are pleased to welcome a new associate editor, Alyson Holland. Alyson comes with experience as editor of another journal, as well as with OJS (open journal systems). We thank the University of Waterloo for providing our OJS online platform, plus essential library staff support.

Editorial Team:

Ellen Desjardins, Editor

University of Waterloo, edesjardins@canadianfoodstudies.ca 
Phil Mount, Associate Editor

Wilfrid Laurier University, pmount@canadianfoodstudies.ca

David Szanto, Associate Editor

University of Gastronomic Sciences, dszanto@canadianfoodstudies.ca

Rod MacRae, Associate Editor

York University, rmacrae@yorku.ca

Wesley Tourangeau, Managing Editor

University of Waterloo, wtourangeau@canadianfoodstudies.ca

Alyson Holland, Associate Editor

McMaster University, aholland@canadianfoodstudies.ca 